\title{
Severe postoperative hemodynamic events after spinal anesthesia a prospective observational study
}

\author{
Edward Bittner ${ }^{1}$, Arielle Butterly ${ }^{3}$, Hooman Mirzakhani ${ }^{3}$, Abhishek Jayadevappa ${ }^{3}$, Theresa MacDonald ${ }^{4}$, Edward George ${ }^{1}$, Ulrich \\ Schmidt $^{2 *}$ and Matthias Eikermann ${ }^{1,4,5}$ \\ Correspondence: uschmidt@partners.org \\ ${ }^{1}$ Harvard Medical School, Director of Critical Care Fellowship Program Department of Anesthesia, Critical Care and Pain Medicine, \\ Massachusetts General Hospital, Boston, MA. \\ ${ }^{2}$ Harvard Medical School, Department of Anesthesia, Critical Care and Pain Medicine, Massachusetts General Hospital, Boston, MA. \\ ${ }^{3}$ Department of Anesthesia, Critical Care and Pain Medicine, Massachusetts General Hospital, Boston, MA. \\ ${ }^{4}$ Patient Care Services, Massachusetts General Hospital, Boston, MA. \\ ${ }^{6}$ Department of Anaesthesia and Intensive Care, Universitaetsklinikum Essen, Essen, Germany.
}

\begin{abstract}
Background: Postoperative hemodynamic adverse severe events (PHASE, severe bradycardia and hypotension) can occur during recovery from spinal anesthesia. The incidence, contributing factors and consequences of PHASE are not well described.

Methods: 232 consecutive patients were included in this prospective observational study. PHASE was defined as a combination of heart rate $<45 \mathrm{bpm}$, and systolic blood pressure $<70 \mathrm{mmHg}$. Correlation analysis was used to identify potential predictors of PHASE and then a multivariate logistic regression model was constructed to evaluate independent predictors of PHASE. PACU lengths of stay between patients with and without PHASE were compared.

Results: Fifteen patients presented with severe hypotension (SBP $64.5 \pm 10.6 \mathrm{mmHg}$ ) and twelve patients with severe bradycardia (heart rate of $40 \pm 5 \mathrm{bpm}$ ), resulting in PHASE in 10 patients. PHASE occurred on average $307 \pm 82 \mathrm{~min}$ after spinal anesthesia with a mean spinal anesthesia level of L1 at the time of PHASE. Insertion of spinal anesthesia in the lateral position (PHASE: $80 \%$, no PHASE: $34 \%$, $\mathrm{p}=0.030)$ as well as morphine dose $(20 \pm 12 \mathrm{mg}$ versus $9 \pm 8 \mathrm{mg}$, respectively $\mathrm{p}=0.011$ ) were found to be independently associated with PHASE. PHASE was associated with a 60 minute increase in median PACU length of stay.

Conclusion: PHASE occurs in about $5 \%$ of patients recovering from spinal anesthesia. The average time for PHASE onset is $307 \pm 82 \mathrm{~min}$. The events are associated with insertion of spinal anesthesia in the lateral compared with sitting position, and with postoperative opioid administration. PHASE during recovery from spinal anesthesia is associated with significantly increased PACU length of stay.
\end{abstract}

Key words: spinal anesthesia, postoperative care, hypotension

\section{Background}

Hypotension and bradycardia are the most common complications associated with spinal anesthesia[1,2], occurring in the intra-operative period due to decreases in systemic vascular resistance and central venous pressure from sympathetic block [1]. Postoperatively, cases of spinal anesthesia associated severe bradycardia have been described to occur up to 5 hours after arrival in the Post Anaesthesia Care Unit (PACU) [3]. To the best of our knowledge, there has been limited description of the epidemiology of severe bradycardia and hypotension during the recovery from spinal anesthesia. The primary study objectives were to determine whether PHASE is associated with increased PACU length of stay.

\section{Methods}

The study was approved by Partners Health Care Institutional Review Board, and conducted in the 32-bed PACU of the Massachusetts General Hospital (MGH) in Boston, Massachusetts, a teaching hospital that serves as a tertiary care referral center, level-1 trauma center, and community hospital for the inner city population of Boston. The PACU staffing model consists of an anesthesia resident on site 24 hours a day, a supervising staff intensivist, a respiratory therapist and a nurse staffing ratio of 1 nurse for every two patients.

\section{Subjects}

We included 232 consecutive ASA physical status 1-3 patients 
Table 1. Patient Demographics

\begin{tabular}{|c|c|c|c|c|}
\hline & $\begin{array}{l}\text { No hemody- } \\
\text { namic event } \\
\mathrm{N}=173(91 \%)\end{array}$ & $\begin{array}{c}\text { Hypoten- } \\
\text { sion } \\
\mathrm{N}=15 \\
(7.9 \%)\end{array}$ & $\begin{array}{l}\text { Bradycardia } \\
\mathrm{N}=12(6.3 \%)\end{array}$ & $\begin{array}{c}\text { PHASE } \\
\mathrm{N}=10(5.2 \%)\end{array}$ \\
\hline \multicolumn{5}{|l|}{ Gender } \\
\hline Age (years) & $67 \pm 11$ & $66 \pm 8$ & $67 \pm 7$ & $67 \pm 4$ \\
\hline Height (inches) & $66 \pm 7$ & $67 \pm 3$ & $68 \pm 4$ & $66 \pm 4$ \\
\hline Weight (kg) & $85 \pm 24$ & $80 \pm 12$ & $82 \pm 11$ & $80 \pm 12$ \\
\hline ASA 1 & 8 & & & \\
\hline ASA 2 & 133 & 1 & 1 & \\
\hline ASA 3 & 32 & 14 & 11 & 10 \\
\hline $\begin{array}{l}\text { Cardiovascular } \\
\text { risk factors }\end{array}$ & $65 \%$ & $56 \%$ & $50 \%$ & $62 \%$ \\
\hline $\begin{array}{l}\text { Beta-blocker } \\
\text { taken in the } \\
\text { morning }\end{array}$ & $32 \%$ & $6.7 \%$ & $8.3 \%$ & $10 \%$ \\
\hline $\begin{array}{l}\text { Other anti- } \\
\text { hypertensives } \\
\text { taken in the } \\
\text { morning }\end{array}$ & $39 \%$ & $33 \%$ & $25 \%$ & $30 \%$ \\
\hline
\end{tabular}

undergoing orthopedic lower limb surgeries arriving in the PACU after spinal anesthesia. Patients with incomplete data sets were excluded from the study and 190 patients with complete data set were included in data analysis (Table 1).

PHASE: A focus group of experienced anesthesiologists and PACU nurses was held to develop a definition of severe postoperative hemodynamic events occurring after spinal anesthesia. Based on the results of this focus group, PHASE was defined as combination of heart rate $<45 \mathrm{bpm}$, and systolic blood pressure $<70 \mathrm{mmHg}$.

\section{Protocol}

Patients were given either 1-2 mg intravenous midazolam as a premedication up to 1 hour prior to spinal placement. Patient care was delivered in the usual manner and was not altered in any way by the investigator. All anesthetics were supervised by board certified staff anesthesiologists. In accord with departmental guidelines, all patients were preloaded with $500-1000 \mathrm{ml}$ of ringers lactate prior to spinal anesthesia which was followed by slow infusion of ringers lactate. Hyperbaric bupivacaine $0.5 \%, 3.6 \pm 1 \mathrm{ml}$ (mean and standard deviation) was injected into L2-3 or L3-4 interspace with the patient in either sitting or lateral position. Standard anesthesia monitoring (electrocardiogram, heart rate, pulse oximetry and oscillometric blood pressure) was used continuously throughout the OR portion of care. Immediately after spinal placement, patients were positioned horizontally and supine as described in the Clincial Prcedures of the Masschusetts General Hospital[5]. Side effects of spinal anesthesia such as nausea and vomiting were documented and treated.
Patients with blood loss of more than $20 \%$ of blood volume were treated with colloids and RBC transfusions. After surgery, patients were transferred to the PACU in the supine position with the head of the bed elevated by 20-30 degrees. Intravenous morphine or hydromorphone were used to provide postoperative analgesia.

\section{Measurements}

Preoperative data collected for patients enrolled in the study included age, weight, height, gender and history of medical illness (diabetes mellitus, hypertension, stroke, arrhythmia, previous myocardial infarction) and medications use (angiotensin converting enzyme inhibitors, adrenergic blockers, calcium antagonists, and diuretics).

Intraoperative data collected on study patients included blood pressure and heart rate, temperature, respiratory rate and oxygen saturation. Severe hypotension was defined as systolic blood pressure less than $70 \mathrm{mmHg}$ which required treatment with a vasopressor. Severe bradycardia was defined as heart rate $<45$ beats per minute which required treatment with ephedrine or atropine. Total duration of surgery was also recorded.

On admission in the PACU the following data were documented by PACU nurses who were not involved in the study: skin temperature (forehead non-contact infrared thermometry), blood pressure, peripheral oxygen saturation, and spread of spinal anesthesia (dermatomal level, determined by pin prick). Standard PACU monitoring was then conducted according to institutional protocol (blood pressure, heart rate, five-channel EKG, peripheral oxygen saturation, Aldrete score). Following admission to the PACU dermatomal level of anesthesia was recorded in 15 minutes intervals until full recovery from spinal anesthesia. All patients were closely monitored in the PACU for the occurrence of PHASE which was documented in the patient's chart and was treated by the PACU attending physician and/or resident, as appropriate. The decision about PACU readiness was made by the PACU nurse and the PACU resident. According to Departmental guidelines, patients were considered ready for discharge after adequate pain control was achieved, they were cardiopulmonary stable, and their sensory residual spinal anesthesia level was below S1. PACU length of stay was defined as the time from PACU admission until PACU discharge readiness. PACU length of stay was recorded by the PACU charge nurse not involved in this study.

\section{Statistical analyses}

Sample size estimation for the study was based on the study hypothesis that PHASE prolongs the PACU length of stay. We used the reports of Ponhold [3] and Butterly [6] to calculate the required sample size. Based on the report of Ponhold [3], describing post operative bradycardia, we expected a PHASE incidence of five to ten percent. We recently reported on the association of postoperative complications and PACU length of stay [6]. Based on these data, we estimated that PHASE might 
Bittner et al. Journal of Anesthesiology and Clinical Science 2012, http://www.hoajonline.com/journals/pdf/2049-9752-1-14.pdf

Table.2 Intraoperative data

\begin{tabular}{lcccc}
\hline & $\begin{array}{c}\text { No hemodynamic } \\
\text { event } \mathbf{N = 1 7 3 ( 9 1 \% )}\end{array}$ & $\begin{array}{c}\text { Hypotension } \\
\mathbf{N = 1 5}(\mathbf{7 . 9 \% )}\end{array}$ & $\begin{array}{c}\text { Bradycardia } \\
\mathbf{N = 1 2 ( 6 . 3 \% )}\end{array}$ & $\begin{array}{c}\text { PHASE } \\
\mathbf{N = 1 0 ( 5 . 2 \% )}\end{array}$ \\
\hline Heart rate prior to induction (bpm) & $74 \pm 11$ & $71 \pm 11$ & $66 \pm 11 *$ & $69 \pm 13$ \\
$\begin{array}{l}\text { Systolic blood pressure prior to } \\
\text { induction (mmHg) }\end{array}$ & $137 \pm 19$ & $130 \pm 21$ & $144 \pm 9$ & $149 \pm 16^{*}$ \\
$\begin{array}{l}\text { Diastolic blood pressure prior to } \\
\text { induction (mmHg) }\end{array}$ & $75 . \pm 123$ & $73 \pm 11$ & $75 \pm 10$ & $76 \pm 11$ \\
Position during spinal bupivacaine & LATERAL-34\% & LATERAL-57\% & LATERAL-67\%* & LATERAL -80\%* \\
administration & SITTING-66\% & SITTING-43\% & SITTTNG-33\% & SITTNG-20\% \\
Bupivacaine dose(mg) & $17.5 \pm 4.5 \mathrm{mg}$ & $18 \pm 1.5 \mathrm{mg}$ & $18.5 \pm 2.5 \mathrm{mg}$ & $18.5 \pm 1.5 \mathrm{mg}$ \\
Intraoperative vasopressor & $73 \%$ & $67 \%$ & $50 \%$ & $50 \%$ \\
Intraoperative bradycardia & 0 & 0 & $8.3 \%$ & 0 \\
Intraoperative antiemetics & $37 \%$ & $46 \%$ & $25 \%$ & $30 \%$ \\
Intraoperative opioids & $73 \%$ & $80 \%$ & $83 \%$ & $80 \%$ \\
\hline
\end{tabular}

${ }^{\star} \mathrm{P}<0.05$ vs. patients without PHASE

be associated with a 60 minute difference in PACU length of stay with a standard deviation of 60 minutes. Accordingly, we expected that a sample size of 190 patients provides a $90 \%$ power to detect an effect of PHASE on PACU length of stay (alpha-error: 5 per cent).

Data analysis was performed using the statistics program SPSS (V 12.0., SPSS Inc., Chicago, III). Demographic data are presented as mean \pm standard deviation for continuous variables and as frequencies and percentages for discrete variables. The dichotomous variable PHASE defined as a combination of heart rate $<45 \mathrm{bpm}$, and arterial blood pressure $<70 \mathrm{mmHg}$, was used as the primary endpoint. The Mann-Whitney $U$ test was used to compare the PACU length of stay in patients with and without PHASE.

The study objectives were to: determine whether PHASE is associated with increased PACU length of stay.

Before start of the study we performed a comprehensive literature review. We also held focus group with experienced anesthesiologists and PACU nurses to discuss potential causes of PHASE. A priori, we identified three categories of variables potentially associated with PHASE:

1) patient associated factors including basic demographics, comorbidities, and ASA physical status

2) intraoperative factors,

3) effects of treatment in the PACU.

Chi square analysis was used to examine the association between PHASE and categorical variables while the $t$ and Mann-Whitney $U$ tests were used to compare normally and non-normally distributed continuous variables respectively between the PHASE and non-PHASE groups. Variables that we considered a-priori to be potential preoperative, intraoperative and postoperative predictors of PHASE included: gender, age, height, weight, preoperative heart rate, preoperative systolic blood pressure, preoperative diastolic blood pressure, preoperative EKG abnormality, preoperative conduc- tion disorder, preoperative use of beta blocker, use of other antihypertensives, patient in lateral position during spinal bupivacaine injection, bupivacaine dose, negative fluid balance, use of vasopressors in OR, use of ephedrine in OR, use of intravenous opioids in OR, episode of bradycardia in OR, PACU heart rate, PACU blood pressure systolic, thoracic level of spinal anesthesia at PACU admission, use of IV narcotics in PACU, long-acting opioid dose (morphine equivalents - assumption: one milligram of hydromorphone is equal to $6 \mathrm{mg}$ of morphine), and IV administration of antiemetics in the PACU. Backward stepwise multiple regression analysis was then used to investigate independent factors with a significant association to PHASE within a multivariate model. Variables with the strongest significant contribution $(p<0.05)$ were regarded as the independent factors associated with the primary endpoint.

\section{Results \& Conclusions \\ Univariate analysis \\ Intraoperative course}

Initial blood pressure was significantly higher in patients with severe hypotension when compared to patients with no events (Table 2). A significantly higher proportion of PHASE patients compared with patients without hemodynamic events $(80 \%$ versus $20 \%, p<0.05)$ received spinal anesthesia in lateral position. There was no significant difference in terms of dose of bupivacaine used in spinal anesthesia and relation between the occurrence of intraoperative bradycardia and hypotension with that of severe bradycardia and hypotension postoperatively (figure 1).

\section{Postoperative course}

Variables measured in the PACU are presented in Table 3. During the observation period in the PACU, all patients had oxygen saturation $>95 \%$ without oxygen mask.

Fifteen patients presented with severe hypotension (SBP 
Bittner et al. Journal of Anesthesiology and Clinical Science 2012,

http://www.hoajonline.com/journals/pdf/2049-9752-1-14.pdf

Table 3. Measurements taken in PACU

\begin{tabular}{|c|c|c|c|c|}
\hline & $\begin{array}{l}\text { No hemodynamic } \\
\text { event } \mathrm{N}=173(91 \%)\end{array}$ & $\begin{array}{l}\text { Hypotension } \\
\mathrm{N}=15(7.9 \%)\end{array}$ & $\begin{array}{l}\text { Bradycardia } \\
\mathrm{N}=12(6.3 \%)\end{array}$ & $\begin{array}{c}\text { PHASE } \\
\mathrm{N}=10(5.2 \%)\end{array}$ \\
\hline HR at admission & $68 \pm 13$ & $65 \pm 12$ & $60 \pm 10^{*}$ & $62 \pm 9.6$ \\
\hline Systolic blood pressure at admission & $119 \pm 18$ & $100 \pm 26^{*}$ & $110 \pm 31$ & $103 \pm 29 *$ \\
\hline \multirow{2}{*}{$\begin{array}{l}\text { Cranial spread of spinal anesthesia at PACU } \\
\text { admission }\end{array}$} & $<\mathrm{T} 12: 72(48 \%)$ & $>T 12: 7(47-\%)$ & $<$ T12: 5(42\%) & $<\mathrm{T} 12: 5(50 \%)$ \\
\hline & $>\mathrm{L} 1: 47(22 \%)$ & $>\mathrm{L} 1: 8(53 \%)$ & >L1: 7(58\%) & >L1: 5(50\%) \\
\hline IV Narcotics in PACU & $80 \%$ & $87 \%$ & $100 \%$ & $100 \%$ \\
\hline Morphine equivalent dose (mg) & $9 \pm 8 \mathrm{mg}$ & $18 \pm 12 \mathrm{mg}^{*}$ & $19 \pm 12 \mathrm{mg} *$ & $20 \pm 12 \mathrm{mg} *$ \\
\hline PACU length of stay & $210 \pm 108$ & $360 \pm 270^{*}$ & $312 \pm 312^{*}$ & $350 \pm 318^{*}$ \\
\hline Time from spinal placement to event & & $304 \pm 94$ & $300 \pm 79$ & $307 \pm 82$ \\
\hline Heart rate (during event) & & $50.22 \pm 18.55$ & $40 \pm 5$ & $41 \pm 5$ \\
\hline \multirow[t]{2}{*}{ Systolic blood pressure during event } & & $64.5 \pm 10.6$ & $69.31 \pm 16.27$ & $62 \pm 8$ \\
\hline & & $<\mathrm{T} 12-4(27 \%)$ & $<\mathrm{T} 12-4(33 \%)$ & $<\mathrm{T} 12-3-30 \%$ \\
\hline \multirow[t]{2}{*}{ Cranial spread at time of event } & & $>$ L1-5(33\%) & $>$ L1-4(33\%) & $>\mathrm{L} 1-4(40 \%)$ \\
\hline & & L5-S4: $6(40 \%)$ & L5-S4: $4(33 \%)$ & L5-S4: $3(30 \%)$ \\
\hline
\end{tabular}

${ }^{\star} \mathrm{P}<0.05$ vs. patients with no events

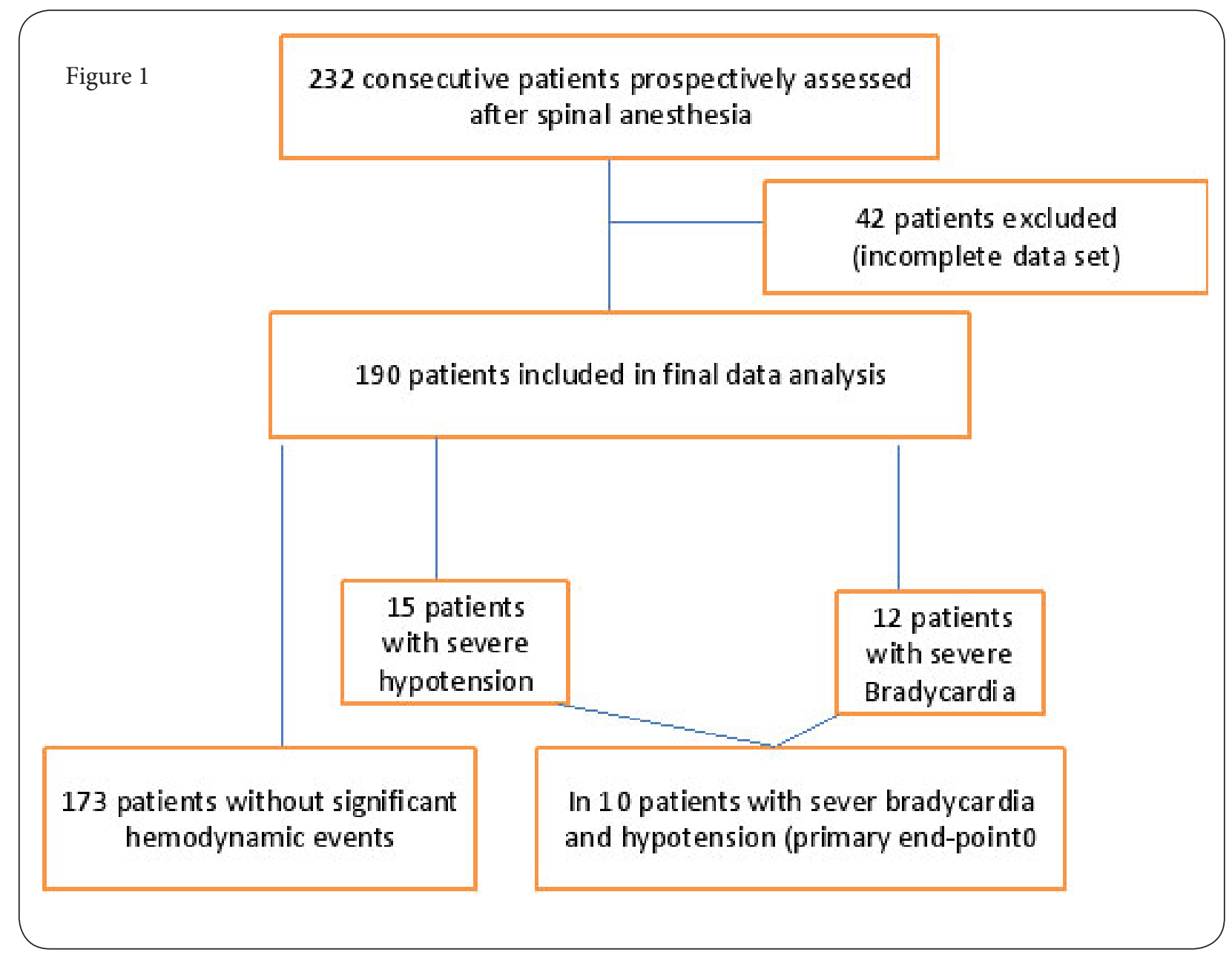




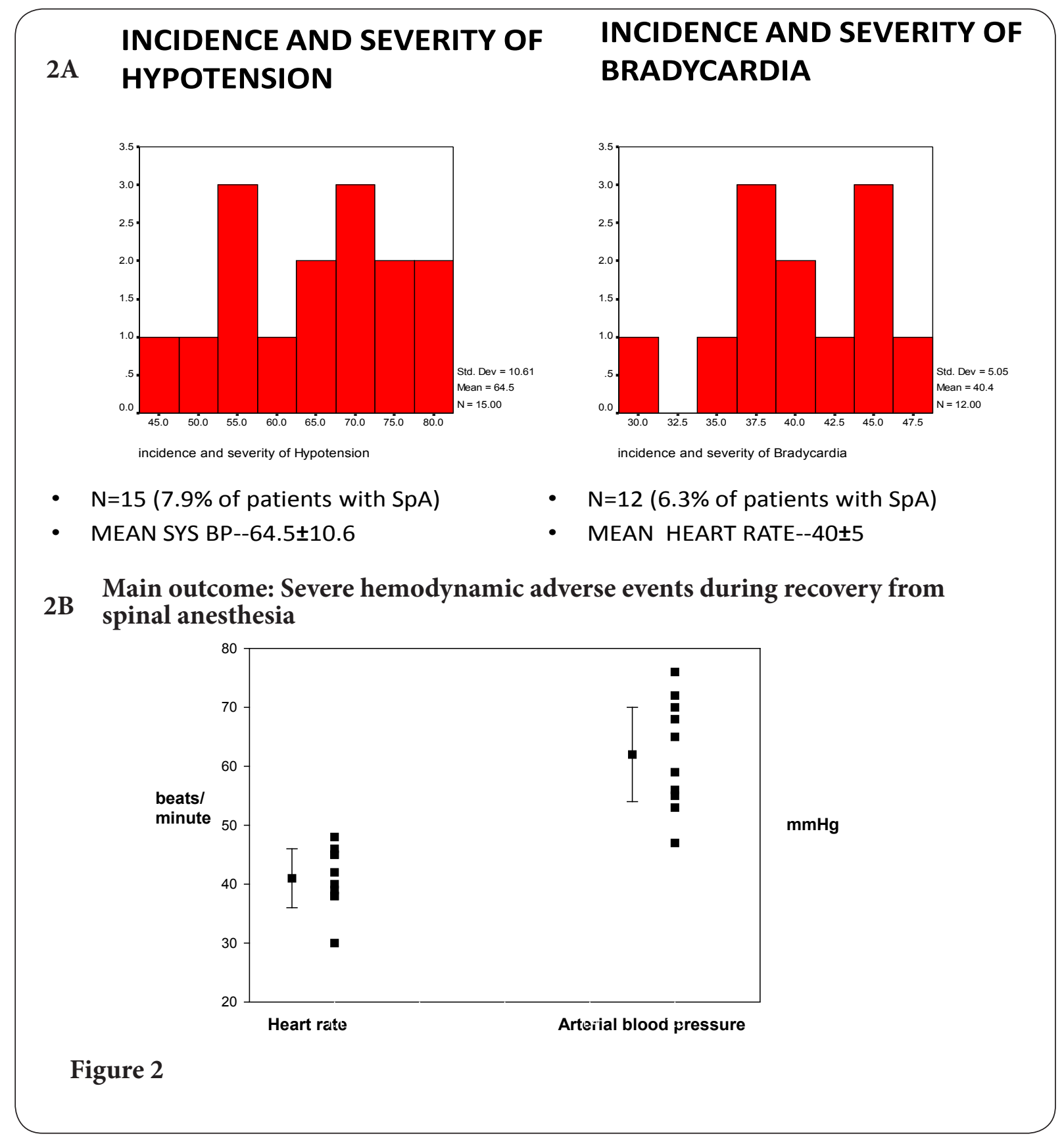

$64.5 \pm 10.6 \mathrm{mmHg}$ ) and twelve patients with severe bradycardia (heart rate of $40 \pm 5 \mathrm{bpm}$ ) (figure 2). Ten patients presented with PHASE, both severe hypotension and bradycardia in the PACU, the main endpoint of our study. Accordingly, the incidence of severe adverse hemodynamic events was 5.2\%. Mean time to occurrence of PHASE was $307 \pm 82 \mathrm{~min}$. Median residual level of spinal anesthesia at PACU admission was $L 1$, without differences between patients with and without PHASE. In the univariate analysis, the following variables were associated with PHASE: Thoracic level of spinal anesthesia at PACU admission $(p<0.0001)$, morphine equivalent dose $(p=0.006)$, total intraoperative fluids $(p=0.031)$, preoperative heart rate $(p=0.046)$, intraoperative vasopressor treatment $(p=0.015)$, and lateral versus sitting position ( $\mathrm{p}=0.038$ ). 


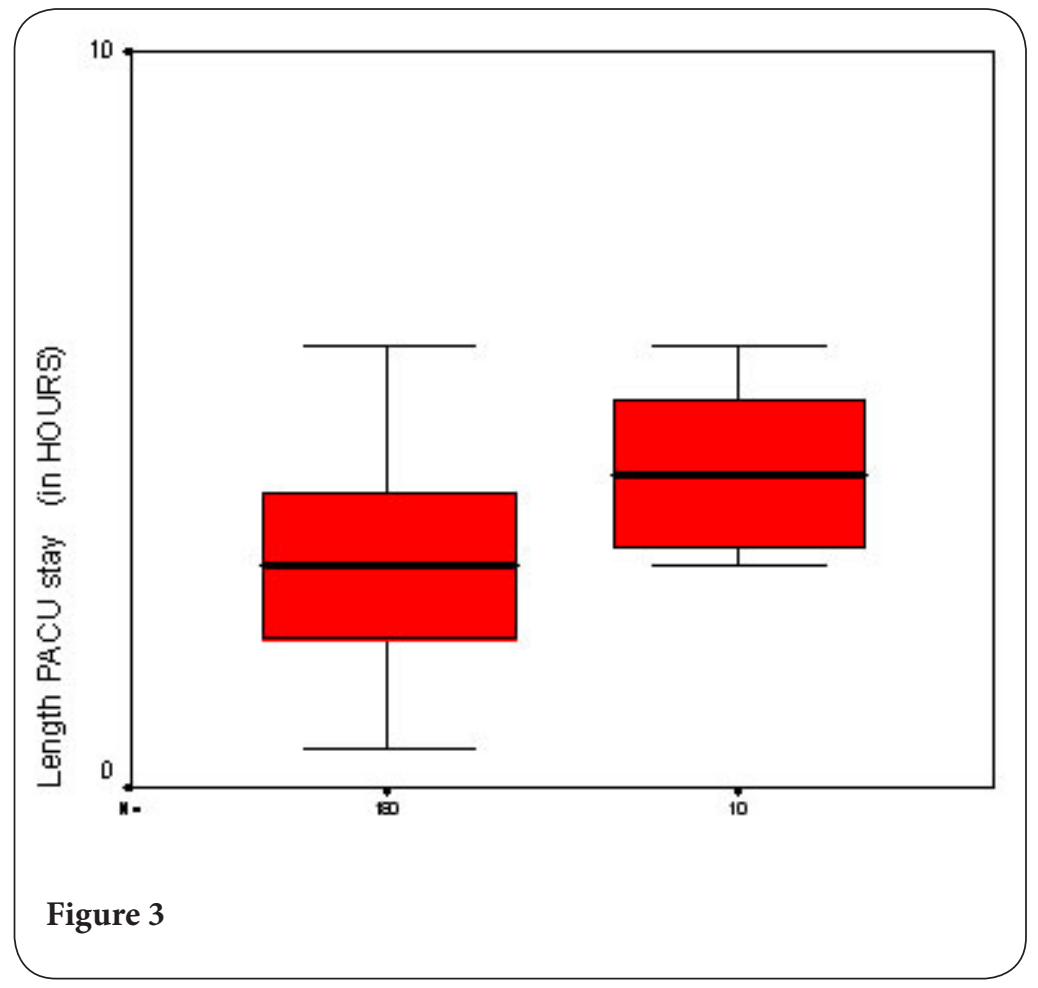

\section{Multivariate analysis}

To identify independent predictors of PHASE, a backward stepwise regression analysis was performed, which initially included the variable found to be associated with PHASE in the univariate analysis: position during insertion of spinal anesthesia (sitting versus lateral flat), preoperative heart rate, intraoperative vasopressors treatment, as well as opioid dose used in PACU.

Insertion of spinal anesthesia in the lateral position (event group: $80 \%$, no-event group: $34 \%, p=0.030$ ) as well as morphine dose $(20 \pm 12 \mathrm{mg}$ versus $9 \pm 8 \mathrm{mg}$, respectively $p=0.011)$ were found to be independently associated with a severe, adverse hemodynamic event.

None of the patients who developed PHASE required cardiopulmonary resuscitation; however all were treated with either atropine, and/or ephedrine.

\section{Effects of severe PHASE on PACU length of stay}

PACU length of stay was significantly longer in patients with adverse severe hemodynamic events (median 240 min, IQR 75 min) compared with patients with no adverse hemodynamic events (180 min, IQR 120, p=0.233; figure 3).

\section{Discussion}

PHASE occurred in $5 \%$ of the patients recovering from spinal anesthesia and was associated with a prolonged PACU length of stay. The combination of bradycardia and hypotension causing hemodynamic instability make PHASE a potential life threatening event. Identifying factors associated with PHASE might help to decrease the incidence.

In our study, the patient's position during spinal placement, as well as the postoperative opioid dose, were independent predictors of PHASE.

Factors contributing to development of hypotension early after spinal anesthesia placement have been well documented $[7,8]$. Acute hemodynamic effects of spinal anesthesia may be explained by sympatholysis, resulting in three major hemodynamic effects: decrease in venous return (in turn influenced by posture, bleeding and inferior vena cava compression), vasodilatation and decreased cardiac output [9].

Bradycardia regularly occurs in patients with higher levels of sympathetic block involving the cardiac accelerator fibers (T1-T4), but this effect was most likely not relevant in our patients. In our patients, the median cranial margin for blockade was L1 and it was substantially lower by the time PHASE occurred (lower lumbar level in $70 \%$ of our PHASE patients). Therefore, even though the uppermost level of spinal anesthesia measured by temperature elevation is up to 6 levels higher compared to the upper limit of sensory blockade [10], it is unlikely that ongoing blockade of cardiac accelerator fibers that can explain the findings of PHASE occurring many hours after placement of spinal anesthesia. We speculate that vagal over-activity, potentially due to a combination of visceral stimulation and opioid effects might have been contributing. Since abdominal visceral structures, such as peritoneum, are innervated by fibers from both limbs 
of the autonomic nervous system, blockade of sympathetic nervous system supply to these organs may result in unopposed parasympathetic nervous system activity. When visceral stimulation, e.g., bladder distension, or visceral pain takes place under these circumstances, parasympathetic afferent transmission may lead to reflexive vagal efferent effects, unrestrained by sympathetic counterbalancing, resulting in bradycardia [11].

The magnitude of hypotension and bradycardia occurring early after spinal administration of local anesthetics should depend on the spread of the sensory block height. In our study, the independent predictor was the position in which the spinal anesthesia was inserted, a variable that is clearly related to the spread of anesthesia. When using hyperbaric bupivacaine, which we did in our study, the onset time to analgesia is shorter in the lateral compared with sitting patients, and hypotension occurs more frequently.

Opioid induced bradycardia [12] may also have contributed to PHASE in part via inhibiting the activity of cardiac vagal neurons $[13,14]$ Furthermore, pre-operative administration of opioids has been shown to be an independent risk factor for intraoperative hypotension in patients undergoing spinal anesthesia [15]. Therefore, we speculate based on our data that the combination of residual (lumbar) neuraxial block and opioid effects contributed to PHASE in our patients.In our study PHASE was associated with lateral positioning during administration of spinal anesthesia was associated with PHASE. Based on this finding lateral positioning during placement of spinal anesthesia might be avoided especially in patients with other identified risk factors for PHASE.

The $6.3 \%$ incidence of bradycardia found in the PACU during recovery from spinal anesthesia is similar to the incidence reported by Ponhold and co-workers [3] despite slightly different definitions Ponhold defined bradycardia as heart rate lower than $50 \mathrm{bpm}$, whereas we used a $45 \mathrm{bpm}$ cut-off point, which we believed to be more clinically meaningful.

The time of occurrence of PHASE was similar to the report of Ponhold and Vincenzi. These authors reported the latest occurrence of severe bradycardia $320 \mathrm{~min}$ after admission to the PACU. Similarly we observed PHASE $455 \mathrm{~min}$ after admission to the PACU. This late occurrence of PHASE makes decisions regarding discharge from the PACU very difficult. The practice guidelines for postoperative care by the American Society of Anesthesiologist's Task Force on Post Anesthetic Care [16] provide little guidance on the best timing for discharge of patient after spinal anesthesia. It states that "Patients should be observed until they are no longer at increased risk for cardio-respiratory depression. A mandatory minimum stay should not be required. Discharge criteria should be designed to minimize the risk of central nervous system or cardio-respiratory depression after discharge". After spinal anesthesia patients are often discharged from the PACU after recovery for motor and sensory function. The concept of orthostatic stability as discharge criteria was introduced by
Alexander [17] and later confirmed by Knoerl et al. [18], these authors reported that using orthostatic blood pressure testing instead of return of motor sensory function could decrease the PACU length of stay to $62 \mathrm{~min}$. While we did not perform orthostatic blood pressure testing it is clinically important that PHASE occurred on average 300 min after placement of spinal anesthesia even in the absence of profound motor block. We conclude based on our data that patients should optimally not be discharged from the PACU until full recovery of sensory and motor function has been documented. Further studies are required to define and test optimal clinical criteria for safe transfer of patients from the PACU.

Severe bradycardia and hypotension can become a life threatening events. None of our patients developed cardiopulmonary arrest; however all required a combination of vasopressors and chronotropic agents. It can be speculated that only the vigilance and early intervention of the PACU staff averted more catastrophic outcomes. Based on the results of this study, our PACU staff now receives periodic refresher training regarding the occurrence and treatment of PHASE in patients' post-spinal anesthesia.

PHASE was associated with a significant increase in PACU length of stay. The magnitude of the effects of PHASE on PACU length of stay is economically meaningful only under the condition that an institution conducts a high volume of spinal anesthesia.

This was a single center study in an academic tertiary care center which limits the ability to generalize the findings to different settings. The study was designed as a prospective observational study which limits the ability to control variables of the study. The intraoperative course including spinal anesthetic was not controlled. It is therefore possible that other factors may influence the occurrence of PHASE. Finally the study's sample size and low incidence of PHASE may have limited our ability to identify risk factors for PHASE especially their contribution in the multivariate analysis.

\section{Conclusion}

In summary, we found that PHASE occurs in 5\% of the patients recovering from spinal anesthesia, and is associated with a prolonged PACU length of stay. In our patients, the patient's position during spinal insertion, as well as the postoperative opioid dose, was independent predictors of PHASE. Further studies are required to define and test optimal clinical criteria for safe transfer of patients after spinal anesthesia.

\section{Competing interests}

The authors declare that they have no competing interests.

\section{Author's contributions}

EB: planned study, analyzed data and drafted the manuscript, AB: collected and analyzed data, HM: collected and analyzed data, EG: planned study and drafted the manuscript, US: planned study, analyzed data and drafted the manuscript, ME: planned study, analyzed data and drafted the manuscript

Funding discloser 
Bittner et al. Journal of Anesthesiology and Clinical Science 2012,

http://www.hoajonline.com/journals/pdf/2049-9752-1-14.pdf

This study was supported by the Department of Anesthesia, Critical Care, and Pain Medicine of the Massachusetts General Hospital. Publication history

Received: 08-June-2012 Revised: 01-Aug-2012

Accepted: 18-Aug-2012 Published: 03-Nov-2012

\section{References}

1. Carpenter, R. L. et al.: Incidence and risk factors for side effects of spinal anesthesia. Anesthesiology 1992, 76(6):906-916. | Article | PubMed

2. Tarkkila, P. J. \& Kaukinen, S.: Complications during spinal anesthesia: a prospective study. Reg Anesth 1991, 16(2):101-106. | PubMed

3. Ponhold, H. \& Vicenzi, M. N.: Incidence of bradycardia during recovery from spinal anaesthesia: influence of patient position. $\mathrm{Br} J$ Anaesth 1998, 81(5):723-726. | Article | PubMed

4. Wilcox, S. R. et al.: Neuromuscular blocking agent administration for emergent tracheal intubation is associated with decreased prevalence of procedure-related complications. Crit Care Med 2012, 40(6):18081813. | Article | PubMed

5. Lewis JM PSM: Clinical Anesthesia Procedures of the Massachusetts General Hospital 8edn: Lippincott Williams \& Wilkins; 2010. | Article | PubMed Abstract | PubMed Full Text

6. Butterly, A. et al.: Postoperative residual curarization from intermediate-acting neuromuscular blocking agents delays recovery room discharge. Br J Anaesth 2010, 105(3):304-309. | Article | PubMed

7. Klasen, J. et al.: Differing incidences of relevant hypotension with combined spinal-epidural anesthesia and spinal anesthesia. Anesth Analg 2003, 96(5):1491-1495, table of contents | Article | PubMed

8. Hartmann, B. et al.: The incidence and risk factors for hypotension after spinal anesthesia induction: an analysis with automated data collection. Anesth Analg 2002, 94(6):1521-1529, table of contents. | Article | PubMed

9. McCrae, A. F. \& Wildsmith, J. A.: Prevention and treatment of hypotension during central neural block. Br J Anaesth 1993, 70(6):672-680. | Article | PubMed

10. Chamberlain, D. P. \& Chamberlain, B. D.: Changes in the skin temperature of the trunk and their relationship to sympathetic blockade during spinal anesthesia. Anesthesiology 1986, 65(2):139-143. | Article | PubMed

11. Riskalla, R. F., Lebowitz, P. W. \& Rubsamen, R. M.: Refractory bradycardia during retrograde pyelography and spinal anesthesia. J Clin Anesth 1988, 1(1):36-38. | Article | PubMed

12. Butterworth, J.: Physiology of spinal anesthesia: what are the implications for management? Reg Anesth Pain Med 1998, 23(4):370-373; discussion 384-377. | PubMed

13. Venkatesan, P. et al.: Glycinergic inputs to cardiac vagal neurons in the nucleus ambiguus are inhibited by nociceptin and mu-selective opioids. J Neurophysiol 2003, 90(3):1581-1588. | Article | PubMed

14. Irnaten, M. et al.: Mu-opioid receptors are located postsynaptically and endomorphin-1 inhibits voltage-gated calcium currents in premotor cardiac parasympathetic neurons in the rat nucleus ambiguus. Neuroscience 2003, 116(2):573-582. | Article | PubMed

15. Tarkkila, P. \& Isola, J.: A regression model for identifying patients at high risk of hypotension, bradycardia and nausea during spinal anesthesia. Acta Anaesthesiol Scand 1992, 36(6):554-558. | PubMed

16. Practice guidelines for postanesthetic care: a report by the American Society of Anesthesiologists Task Force on Postanesthetic Care. Anesthesiology 2002, 96(3):742-752. | Article | PubMed

17. Alexander, C. M. et al.: New discharge criteria decrease recovery room time after subarachnoid block. Anesthesiology 1989, 70(4):640-643. | Article | PubMed
18. Knoerl, D. V., McNulty, P., Estes, C. \& Conley, K.: Evaluation of orthostatic blood pressure testing as a discharge criterion from PACU after spinal anesthesia. J Perianesth Nurs 2001, 16(1):11-18. | Article | PubMed

\section{Citation:}

Schmidt U, Bittner E, Butterly A, Mirzakhani H, Jayadevappa A, MacDonald T, George E and Eikermann M: Severe postoperative hemodynamic events after spinal anesthesia a prospective observational study. journal of Anesthesiology and Clinical Science 2012, 1:14. http://dx.doi.org/10.7243/2049-9752-1-14 